whisper Lues' discusses the management of positive serology for syphilis in the geriatric patient. 'Insight and hindsight' is a discussion on the value of routine sigmoidoscopy. 'Pills and their ills' is on analgesic nephropathy, and 'All that glitters is not diagnostic gold' refers to the various types of fluorescent antibody staining appearances in systemic lupus erythematosus and allied disorders. This monograph is strongly recommended for postgraduates of all disciplines. It is light hearted, educative and a joy to read.

\section{Controlled Therapeutic Trials in Cancer. UICC Information Office. List of Registered Trials}

Edited by R. Flamant and C. Fohanno. UICC Technical Report Series. Vol. 32. Pp. xxix +684 . International Union against Cancer, Geneva 1978. Sw. Fr. 28.00.

The proliferation of trials of therapy in cancer throughout the world has made it necessary to keep a register. This volume lists under each malignancy the current trials of therapy, briefly notes the type of study and gives the author and the institute responsible.

The International Union Against Cancer, U.I.C.C., is to be congratulated on its brave attempt at this impossible task. The value of the book lies in its information on as yet unpublished trials and allows workers on a particular problem to be aware of what is being done elsewhere. Unfortunately many trials are not notified to the Office and consequently this limits the value of the publication. In time it is hoped that increasing international co-operation will improve this and as long as it does not make the book impossibly large it will be a useful addition to the information services on cancer.

\section{Dermatology in Internal Medicine}

By Sam Shuster. Pp. 290. Oxford Medical Publications. Oxford University Press, Oxford, New York and Toronto, 1978. Paperback $£ 5.95$.

Skin diseases are difficult to describe to the uninitiated and the relatively uninterested, it is disappointing, therefore, that this book bears no illustrations and little space is given to histopathology. The chapter on lympho-reticular disorders contains no hint of the $T$ and $B$ cell revolution.

The balance of some of the subjects treated is not altogether appropriate so that the mechanism of the itch is allocated only one fifth of the space given to melanization. Perhaps the approach to internal medicine should have been through fundamental mechanisms and their disorders rather than in the manner of the pedestrian texts of old. The ramifications of haemorheology are scarcely touched upon in 'Vasculitis' and the non-pathogenic arcane classifications are reiterated and not castigated. The potential of the patient's genetic diathesis as a determinant of his disease type, for example the relationship between psoriasis and HLA B27, could have been more fully developed.

The headings sometimes fail to reflect the precise importance and relationship of the subjects so that constitutional seborrhoeic eczema is given as a subheading of acquired contact eczema. The references terminate only some sections, and appear to be of a somewhat random nature.

This book could be useful in a small way for the established physician in internal medicine who carries in his mind's eye a picture of the spots; but it is a bit of a 'curate's egg' for the M.R.C.P. candidate with only a cursory integration into the established fields of medicine, biochemistry, and immunology. It is disappointingly synoptic and straightforward and unlikely to provide 'an integrated conceptual framework' of dermatology, perhaps the most difficult subject in medicine.
Diabetes Mellitus

By M. I. Drury. Pp. viii +125 . Blackwell Scientific Publications, Oxford, 1979. £4.25.

This is a short and practical text book on current concepts of aetiology and management of diabetes. It is written by a physician of wide experience and authority in this subject and the advice he gives is practical and to the point. The book should prove particularly valuable to the newly qualified doctor faced with the care of diabetics as well as the general physician who has had no particular experience in the management of this common disorder.

The earlier chapters on the aetiology and classification of diabetes epitomize the present attitudes to this changing subject and should do much to clarify the confusion that must arise from perusal of the vast literature available. The book is up to date, easy to read and contains useful references after each chapter for further reading.

It can be warmly recommended and should find a place on every medical ward that has to deal with diabetes.

\section{Hospital-Associated Infections in the Compromised Host}

Edited by G. P. Bodey and V. Rodriguez. Pp. xi +263 , illustrated. Marcel Dekker, New York and Basle, 1979. Sw.Fr. 74.00

This is the second book in a series of 4 on hospital-associated infections. There are 8 authors who between them have contributed 6 sections.

The opening section is a comprehensive but concise review of the causes and the assessment of the immunosuppressed state and the immunological defects in a number of common tumours. This will be of use to the non-immunologist. The shortest section (on surgical procedures in diagnosis of infection) is precise and has useful recommendations paro ticularly concerning interstitial pneumonitis. Sections on the clinical course, the treatment of infections and infections with unusual organisms contain much factual detail as weli as some repetition and would have benefited from being shorter, supplemented as each section is by extensive references. The section on protective isolation cannot be considered a review as it mainly consists of results already reported in 3 of the author's publications.

The book does not read easily in many places and cannot be recommended as a textbook but would provide a useful reference source.

\section{Immunodiagnosis of Cancer. Part 1. Immunology Series Volume 9}

Edited by Ronald B. Herberman and K. Robert MCINTIRE. Pp. xvi + 702, illustrated. Marcel Dekker, New York and Basel, 1979. Sw.Fr. 130.00.

This book is part of a series on Immunology and is only Part (despite 700 pages) on the subject of immunodiagnosis of cancer. The editors state their intention of reviewing the field for the tumour immunologist and the clinician, and they have spared no efforts to achieve this end. After dealing with the problems of immunodiagnosis in general, a very large portion of the book is devoted to the detection of circulating tumourassociated markers-in particular $\alpha$-fetoprotein, carcinoembryonic antigen and human chorionic gonadotrophin The methodology is critically reviewed and it says a great deal for the editors that there is little needless repetition in any of these sections. The clinician and the tumour immunologist will find a great deal of interest in this section. The last section on detection of tumour-associated and other antigens on tumour cells contains excellent reviews of the state of these studies in acute leukaemia, melanoma, ovarian and other cancers, and will probably appeal more to the clinician. This volume is very well documented and one awaits Part 2 with interest. 